\title{
A Scalar Compromise Equilibrium for N-Person Prescriptive Games
}

\author{
H. W. Corley ${ }^{*}$, Surachai Charoensri ${ }^{2}$, Narakorn Engsuwan ${ }^{3}$ \\ ${ }^{1}$ Center on Stochastic Modeling, Optimization, \& Statistics (COSMOS), The University of Texas at Arlington, \\ Arlington, TX, USA \\ ${ }^{2}$ Kasikorn Bank PCL, Bankgok, Thailand \\ ${ }^{3}$ IMSE Department, The University of Texas at Arlington, Arlington, TX, USA \\ Email: ${ }^{\text {corley@uta.edu }}$
}

Received 17 June 2014; revised 22 July 2014; accepted 5 August 2014

Copyright (C) 2014 by authors and Scientific Research Publishing Inc.

This work is licensed under the Creative Commons Attribution International License (CC BY). http://creativecommons.org/licenses/by/4.0/

(c) (i) Open Access

\begin{abstract}
A scalar equilibrium (SE) is defined for n-person prescriptive games in normal form. When a decision criterion (notion of rationality) is either agreed upon by the players or prescribed by an external arbiter, the resulting decision process is modeled by a suitable scalar transformation (utility function). Each n-tuple of von Neumann-Morgenstern utilities is transformed into a nonnegative scalar value between 0 and 1 . Any n-tuple yielding a largest scalar value determines an SE, which is always a pure strategy profile. SEs can be computed much faster than Nash equilibria, for example; and the decision criterion need not be based on the players' selfishness. To illustrate the SE, we define a compromise equilibrium, establish its Pareto optimality, and present examples comparing it to other solution concepts.
\end{abstract}

\section{Keywords}

Game Theory, Equilibria, Scalar Equilibrium, Compromise Equilibrium, Scalar Transformation, Prescriptive Analysis

\section{Introduction}

Game theory is the study of strategic interactions among agents called players. Ultimately it involves a solution concept to describe, predict, or prescribe the choices of these players [1]. Modern game theory [2] [3] is predominantly noncooperative and assumes that any joint rational action by the players must necessarily be a Nash Equilibrium (NE) [4] [5]. In other words, rational players are assumed to be selfish. They act in their individual self-interest in the sense that each player considers his best responses to the possible joint actions of the other

${ }^{*}$ Corresponding author. 
players. The result is that no player can improve his expected payoff in an NE by unilaterally changing his pure or mixed strategy. Various refinements of the NE (see [2] [3], for example) have been proposed, yet the NE does not suffice for all strategic interactions. Social dilemmas [6] [7] illustrate that selfish behavior may conflict with group interests. For example, in Prisoner's Dilemma each player can do better by cooperating.

In this paper we consider one-shot, n-person games in normal form. A player is considered rational if his strategy choices are consistent with some underlying decision-making criterion. For example, instead of being greedy, a player may be satisfied with a certain level of payoff. To provide a theoretical framework for such cases, we define a scalar equilibrium (SE) in which a scalar transformation modeling the decision criterion assigns scalar values in $[0,1]$ to the outcome of each joint action of the players. An SE is a joint action maximizing these scalar values.

SEs address three problematic areas of noncooperative game theory.

1) SEs do not require that rationality be defined by selfish behavior.

2) An SE consists of pure strategies for each player, as opposed to mixed strategies that are difficult to interpret and implement [8].

3) NEs are difficult to compute [9], while SEs can be quickly obtained by simply finding the maximum of a finite number of scalar values.

SEs are particularly applicable (a) when all players have the same notion of rationality or (b) when an external arbiter dictates each player's strategy according to some predetermined decision criteria. In case (a) the SE is descriptive or predictive- or possibly normative in the sense that it suggests actions for the players. In case (b), which is emphasized here, the SE is prescriptive.

The paper is organized as follows. In Section 2 we formally define the SE. In Section 3 we illustrate SEs with the compromise equilibrium (CE) and establish its Pareto optimality. In Section 4 we present some examples of two-person games and compare the CE with other solution concepts. Finally, in Section 5 we offer conclusions and discuss future research.

\section{Scalar Equilibrium}

Let $\Gamma=\left\langle N,\left(S_{i}\right)_{i \in N},\left(u_{i}\right)_{i \in N}, A\right\rangle$ denote an n-person, one-shot prescriptive game in normal form, where $N=\{1, \cdots, n\}$ is the set of players, $S_{i}$ is the finite set of pure strategies for player $i, u_{i}(s)$ is the von Neumann-Morgenstern (VMN) utility of player $i$ for a pure strategy profile $s=\left(s_{1}, \cdots, s_{n}\right) \in S=\prod_{i=1}^{n} S_{i}$ for all players, and $u(s)=\left(u_{1}(s), \cdots, u_{n}(s)\right) . A$ is an arbiter who assigns strategies for the players in their one-shot.

$A$ need not be a person. The arbiter could be a common decision criterion applied by the $\mathrm{n}$ players. The arbiter could be a licensing agreement for the licensees of a patent, for example. It could be a computer algorithm for making real-time decisions on a website where the players have agreed to its terms and conditions. In the current regulatory spirit, it could also be a policy imposed by a national governmental agency on some segment of the population. In other words, an arbiter is a prescriptive agent.

Regardless, A's decision criterion for assigning strategies can be represented by an ordinal utility function $T_{A}: u(S) \rightarrow R^{1}$ (see [10] [11]) that induces a preference relation $\leq_{A}$ on $u(S)$. For $s^{\prime}, s^{\prime \prime} \in S$ we write $u\left(s^{\prime}\right)<_{A} u\left(s^{\prime \prime}\right)$ if $T_{A}\left[u\left(s^{\prime}\right)\right]<T_{A}\left[u\left(s^{\prime \prime}\right)\right], u\left(s^{\prime}\right)=_{A} u\left(s^{\prime \prime}\right)$ if $T_{A}\left[u\left(s^{\prime}\right)\right]=T_{A}\left[u\left(s^{\prime \prime}\right)\right]$, and $u\left(s^{\prime}\right) \leq_{A} u\left(s^{\prime \prime}\right)$ if either $u\left(s^{\prime}\right)<_{A} u\left(s^{\prime \prime}\right)$ or $u\left(s^{\prime}\right)=_{A} u\left(s^{\prime \prime}\right)$. A is said to be indifferent between $s^{\prime}$ and $s^{\prime \prime}$ if $T_{A}\left[u\left(s^{\prime}\right)\right]=T_{A}\left[u\left(s^{\prime \prime}\right)\right]$. Thus $A$ may be considered rational in the sense that $\leq_{A}$ is complete and transitive.

Definition 2.1. The pure strategy profile $s^{*}$ is an SE for $\Gamma$ if and only if $T_{A}[u(s)] \leq T_{A}\left[u\left(s^{*}\right)\right]$ for all $s \in S$. Thus $s^{*}$ is an SE if it maximizes the scalar composite function $T_{A}$ ou over $S$.

If $\Gamma$ has multiple SEs resulting from ties in the maximization of Definition 3.1, it is assumed that $A$ will choose one $s^{*}$ from the SEs by some further mechanism.

\section{Compromise Equilibrium}

The compromise equilibrium CE for $\Gamma$ is now presented as an example of an SE. Since $S_{i}$ is a finite set of 
pure strategies for each player, $u_{i}(s)$ is bounded. We write $M_{i}=\max _{s \in S} u_{i}(s)$ and $m_{i}=\min _{s \in S} u_{i}(s)$ for $i=1, \cdots, n$. Now for all $s \in S$ define the transformation $T_{\mathrm{A}}: u(S) \rightarrow R^{1}$ by

$$
T_{A}[u(s)]=\prod_{i=1}^{n} \frac{u_{i}(s)-m_{i}+1}{M_{i}-m_{i}+1} .
$$

Note that $0<T_{A}[u(s)] \leq 1$ for all $s \in S$. The number 1 in the numerators of Equation (1) prevents $T_{A}[u(s)]$ from being 0 for an $s \in S$ for which $u_{i}(s)=m_{i}$ for some $i$, while the 1 in the denominators prevent a division by 0 for an $i$ for which $m_{i}=M_{i}$.

The intuition behind Equation (1) is explained as follows. If we maximize the function $f\left(z_{1}, \cdots, z_{n}\right)=\prod_{i=1}^{n} z_{i}$ over the region $0<z_{i} \leq 1, i=1, \cdots, n$, the maximum is given by $z_{i}^{*}=1, i=1, \cdots, n$. Similarly, maximizing Equation (1) yields a CE $s^{*}$ for which the terms $\frac{u_{i}\left(s^{*}\right)-m_{i}}{M_{i}-m_{i}}$ are large and close in value for those $i$ for which $M_{i}>m_{i}$. Otherwise $u_{i}\left(s^{*}\right)=M_{i}=m_{i}$. Thus if an arbiter $A$ applies Equation (1), the players with $M_{i}>m_{i}$ will receive payoffs in roughly the same percentile of their payoff ranges. If maximizing $T_{A}[u(s)]$ is the decision criterion for all players (with ties broken by some further mechanism), the outcome can be construed as an equitable compromise between the players' selfishness and unselfishness. A CE, however, differs substantially from the Rabin's fairness equilibrium [12] for two players and from other notions of fairness as presented in [13].

Any CE is also a Pareto optimum [14] of $u(S)$, which is essential for a solution concept to an n-person game [15]. For example, a pure NE not Pareto optimal cannot be a joint rational solution since an alternate strategy profile can improve some players' payoffs without diminishing anyone's.

Definition 3.1. The pure strategy profile $s^{\prime \prime} \in S$ dominates $s^{\prime} \in S$ if and only if $u_{i}\left(s^{\prime}\right) \leq u_{i}\left(s^{\prime \prime}\right)$ for $i=1, \cdots, n$ and $u_{j}\left(s^{\prime}\right)<u_{j}\left(s^{\prime \prime}\right)$ for some $j$. A pure strategy profile $s^{*} \in S$ is Pareto optimal for $\Gamma$ if $s^{*}$ is not dominated by any $s \in S$.

Lemma 3.2. For any $s^{\prime}, s^{\prime \prime} \in S$, if $s^{\prime \prime}$ dominates $s^{\prime}$, then $T_{A}\left[u\left(s^{\prime}\right)\right]<T_{A}\left[u\left(s^{\prime \prime}\right)\right]$.

Proof. Let $s^{\prime}, s^{\prime \prime} \in S$, and suppose that $s^{\prime \prime}$ dominates $s^{\prime}$. From Definition 3.1 it follows that $u_{i}\left(s^{\prime}\right) \leq u_{i}\left(s^{\prime \prime}\right)$ for all $i=1, \cdots, n$ and $u_{j}\left(s^{\prime}\right)<u_{j}\left(s^{\prime \prime}\right)$ for some index $j$. Thus for $i=1, \cdots, n$

$$
0<\frac{u_{i}\left(s^{\prime}\right)-m_{i}+1}{M_{i}-m_{i}+1} \leq \frac{u_{i}\left(s^{\prime \prime}\right)-m_{i}+1}{M_{i}-m_{i}+1},
$$

and for $j$

$$
0<\frac{u_{j}\left(s^{\prime}\right)-m_{j}+1}{M_{j}-m_{j}+1}<\frac{u_{j}\left(s^{\prime \prime}\right)-m_{j}+1}{M_{j}-m_{j}+1} .
$$

Together Equation (2) and Equation (3) give

$$
\prod_{i=1}^{n} \frac{u_{i}\left(s^{\prime}\right)-m_{i}+1}{M_{i}-m_{i}+1}<\prod_{i=1}^{n} \frac{u_{i}\left(s^{\prime \prime}\right)-m_{i}+1}{M_{i}-m_{i}+1},
$$

so $T_{A}\left[u\left(s^{\prime}\right)\right]<T_{A}\left[u\left(s^{\prime \prime}\right)\right]$.

Theorem 3.3. If $s^{*}$ is a CE for $\Gamma$, then $s^{*}$ is Pareto optimal for $\Gamma$.

Proof. Let $s^{*}$ be a CE for $\Gamma$. We prove the contrapositive. If $s^{*}$ is not Pareto optimal, there exists $s^{\prime} \in S$ that dominates $s^{*}$. From Lemma 3.2 it follows that $T_{A}\left[u\left(s^{*}\right)\right]<T_{A}\left[u\left(s^{\prime}\right)\right]$, so $s^{*}$ is not a CE for $\Gamma$. 


\section{Examples}

We now present two examples for $n=2$ to illustrate the CE. For $n>2$, calculations using Equation (1) are similar. In the examples, we compare the CE to any pure NE, where each player's action selfishly maximizes his payoff for the action of the other player. We also compare CEs to any mutual-max outcome [12], where each player unselfishly maximizes the payoff for other player's action. Finally, we compare CEs to maximin outcomes [2] in which each player's action maximizes his minimum payoff resulting from the actions of the other players.

Example 4.1. Consider the Prisoner's Dilemma (PD) payoff matrix of Figure 1, where D denotes the action "Defect" and C denotes "Cooperate". The pure NE is (D, D), the mutual-max outcome is (C,C), and the maximin outcome is (D, D). The matrix of values calculated from Equation (1) is shown in Figure 2, from which the Pareto optimum (C, C) is the unique CE. Note that the mutual-max outcome and the CE are the same.

Example 4.2. Consider now the discoordination game [16] given by Figure 3. Players 1 and 2 approach each other. Player 1's incentive is to veer right (R) or left (L) in the opposite direction from Player 2's move. However, Player 2's incentive is to encounter Player 1. There is no pure NE or mutual-max outcome for this game. The maximin outcome is (R, R). The CE scalar values are shown in Figure 4, with the unique CE being the Pareto optimum (R, R). The maximin outcome is the same as the CE.

\section{Conclusions}

In this paper the general notion of a scalar equilibrium SE is defined for an n-person, one-shot game in normal form. The advantages of an SE include flexibility in the decision criteria for decision makers, the selection of pure strategies for the players, and the speed of computing an SE. An SE is most applicable when the games are prescriptive, i.e., when an arbiter $A$ assigns the players' actions for their one shot.

The compromise equilibrium CE is presented here as a special case of an SE. Significantly, a CE is also a Pareto optimal pure strategy profile for the VNM utilities. It may be interpreted as a reasonable tradeoff of payoffs

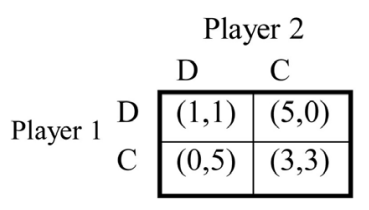

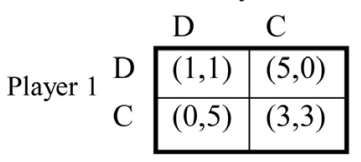

Figure 1. PD payoff matrix.

Player 2

\begin{tabular}{|c|c|c|}
\hline \multirow{3}{*}{ Player 1} & D & $\mathrm{C}$ \\
\hline & 0.111 & 0.167 \\
\hline & 0.167 & 0.667 \\
\hline
\end{tabular}

Figure 2. Scalar values for Figure 1.

Player 2

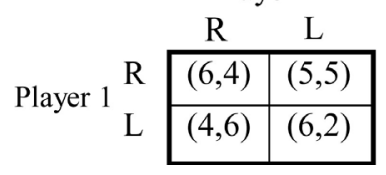

Figure 3. Discoordination game.

$$
\begin{array}{ccc}
\multicolumn{2}{c}{} & \multicolumn{2}{c}{\text { Player 2 }} \\
\multicolumn{1}{c}{\text { R }} & \multicolumn{1}{c}{\text { L }} \\
\cline { 2 - 3 } \text { Player 1 } & 0.600 & 0.533 \\
\cline { 2 - 3 } & 0.633 & 0.200 \\
\cline { 2 - 3 } & &
\end{array}
$$


imposed on the players by the arbiter $A$. Future research should explore other SEs. For example, one might define a transformation $T_{A}[u(s)]$ to obtain an SE approximating a pure NE when one does not exist.

\section{References}

[1] Aumann, R. (1985) What Is Game Theory Trying to Accomplish? In: Arrow, K. and Honkapohja, S., Eds., Frontiers of Economics, Basil Blackwell, Oxford, 5-46.

[2] Maschler, M., Solan, E. and Zamir, S. (2013) Game Theory. Cambridge University Press, Cambridge. http://dx.doi.org/10.1017/CBO9780511794216

[3] Myerson, R. (1991) Game Theory: Analysis of Conflict. Harvard University Press, Cambridge.

[4] Nash, J. (1950) Equilibrium Points in N-Person Games. Proceedings of the National Academy of Sciences, 36, 48-49. http://dx.doi.org/10.1017/CBO9780511794216

[5] Nash, J. (1951) Non-Cooperative Games. The Annals of Mathematics, 54, 286-295. http://dx.doi.org/10.2307/1969529

[6] Poundstone, W. (2011) Prisoner’s Dilemma. Random House, New York.

[7] Beckenkamp, M. (2006) A Game-Theoretic Taxonomy of Social Dilemmas. Central European Journal of Operations Research, 14, 337-353. http://dx.doi.org/10.1007/s10100-006-0008-5

[8] Rubinstein, A. (1991) Comments on the Interpretation of Game Theory. Econometrica, 59, 909-924. http://www.jstor.org/stable/2938166 http://dx.doi.org/10.2307/2938166

[9] Daskalakis, C., Goldberg, P. and Papadimitriou, C. (2009) The Complexity of Computing a Nash Equilibrium. SIAM Journal on Computing, 39, 195-209. http://dx.doi.org/10.1145/1461928.1461951

[10] Barbera, S., Hammond, P. and Seidl, C. (1999) Handbook of Utility Theory: Volume 1 Principles. Springer, New York.

[11] Barbera, S., Hammond, P. and Seidl, C. (2004) Handbook of Utility Theory: Volume 2 Extensions. Springer, New York. http://dx.doi.org/10.1007/978-1-4020-7964-1

[12] Rabin, M. (1993) Incorporating Fairness into Game Theory and Economics. The American Economic Review, 83, 1281-1302.

[13] Korth, C. (2009) Fairness in Bargaining and Markets. Springer-Verlag, New York. http://dx.doi.org/10.1007/978-3-642-02253-1

[14] Ehrgott, M. (2005) Multicriteria Optimization. Springer-Verlag, New York.

[15] Harsanyi, J. and Selten, R. (1988) A General Theory of Equilibrium Selection in Games. MIT Press, Cambridge.

[16] Cooper, R. (1998) Coordination Games. Cambridge University Press, Cambridge. 
Scientific Research Publishing (SCIRP) is one of the largest Open Access journal publishers. It is currently publishing more than 200 open access, online, peer-reviewed journals covering a wide range of academic disciplines. SCIRP serves the worldwide academic communities and contributes to the progress and application of science with its publication.

Other selected journals from SCIRP are listed as below. Submit your manuscript to us via either submit@scirp.org or Online Submission Portal.
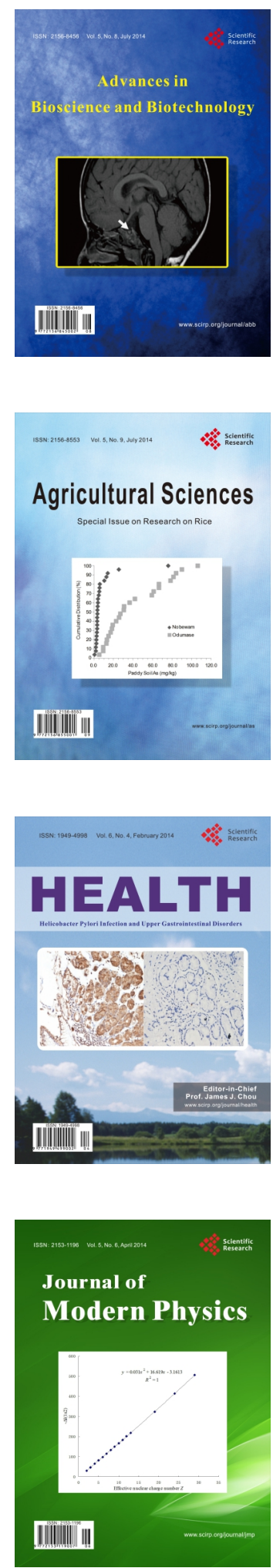
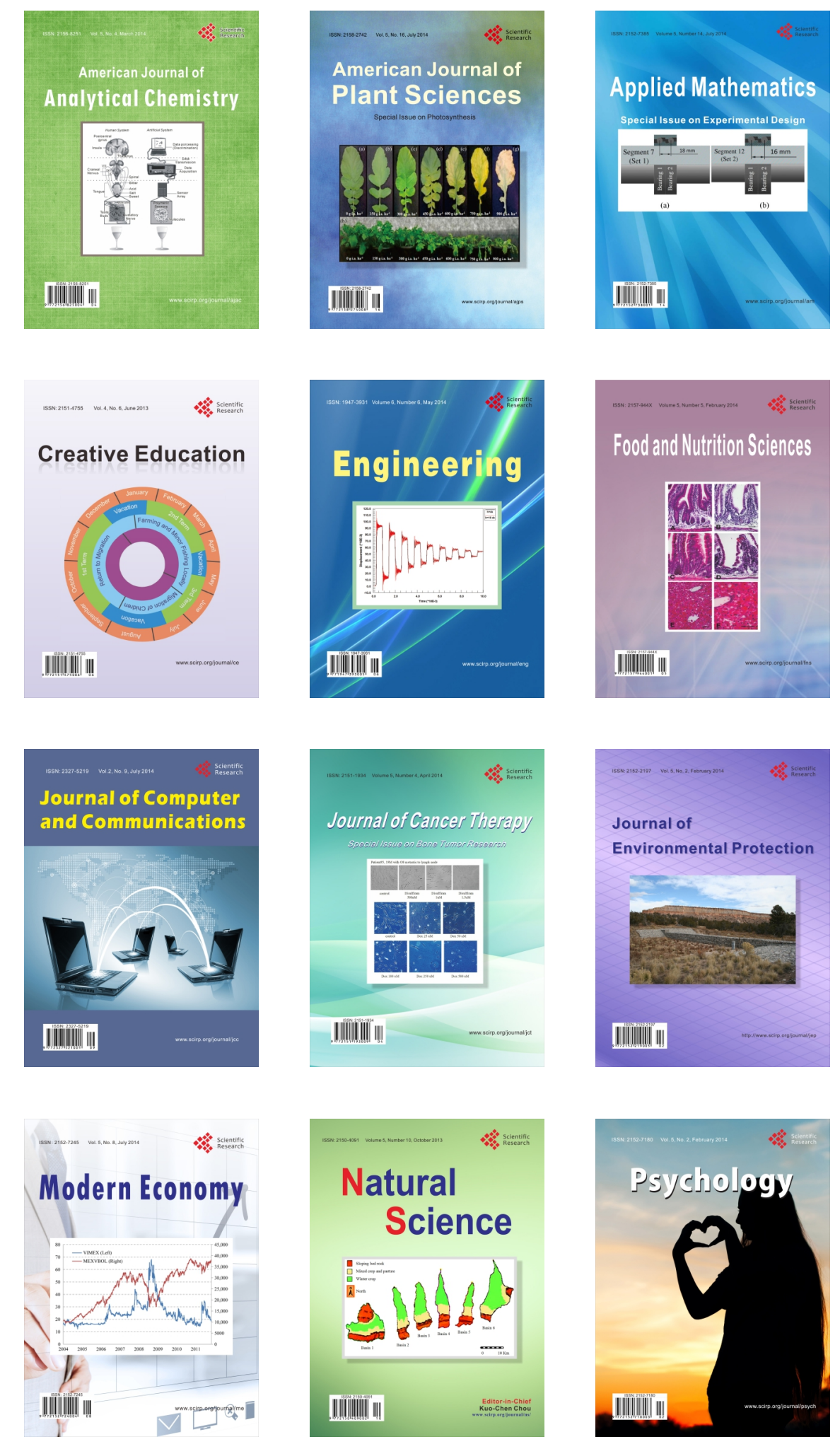